hep-ph/0002119

SMU-HEP-00-05

\title{
SIMP (Strongly Interacting Massive Particle) Search㠹
}

\author{
Vigdor L. Teplitz, ${ }^{1}$ Rabindra N. Mohapatra, ${ }^{2}$ Fred Olness, ${ }^{1}$ and \\ Ryszard Stroynowski ${ }^{1}$ \\ ${ }^{1}$ Department of Physics, Southern Methodist University, Dallas, TX 75275 \\ ${ }^{2}$ Department of Physics, University of Maryland, College Park, MD 20742.
}

\section{Introduction}

Strongly Interacting Massive Particle (SIMPS), by which we will always mean neutral, stable SIMPs, are of current interest for at least three reasons:

- They could be a dark matter constituent as suggested some time ago by Dover, Gaisser and Steigman [1] and by Wolfram [2]. Starkman et al.,[3] show SIMPs would be restricted to rather narrow mass ranges if they were to exhaust $\Omega=1$. We will not make this assumption and will consider SIMPs outside the regions allowed by the analysis of ref. [3].

- It is possible that the lightest SUSY particle (LSP) is strongly interacting and hence, if R-parity is conserved, would form a colorless SIMP. Possibilities, such as a $\tilde{g} g$ bound state are discussed in ref. [4].

- An explanation of the ultra high energy cosmic ray events (UHECRs) proposed by Farrar, Kolb and co-workers [5] is that they are due to interactions of SIMPs

\footnotetext{
*Presented by Vigdor L. Teplitz.

${ }^{\dagger}$ To appear in the proceedings of the International Conference On Orbis Scientiae 1999: Quantum Gravity, Generalized Theory Of Gravitation And Superstring Theory Based Unification, 16-19 December 1999, Fort Launderdale, Florida.
} 
with a mass below $50 \mathrm{GeV}$ and a cross section for interactions with nucleons on the order of a (few) millibarns.

This summary will review two laboratory experiments that might detect SIMPs. For more detail, the reader is encouraged to examine with care ref. [6] and the paper on which it is based [7].

In Section 2 we consider the possibility of finding SIMPs bound in ordinary nuclei by searching for anomalously heavy isotopes of high-Z nuclei. It is a pleasure to note that the accelerator mass spectrometry (AMS) group at Purdue is in the process of performing the experimentf suggested in ref. [6]. In Section 3, we address the extent to which production and detection of SIMP-anti-SIMP $(S \bar{S})$ pairs might be performed at the Tevatron.

Our results, in brief, are that the AMS experiment should be sensitive to SIMPs over a wide range of parameter space: $\left(\sigma_{S N}, M_{S}\right)$, where $M_{S}$ is the SIMP mass and $\sigma_{S N}$ is its cross section for scattering off nucleons. The Tevatron, on the other hand is likely only to produce and to detect SIMPs in a much more restricted range, but one that includes much of the mass range for which the SIMP could be the UHECR explanation. It would be only fitting, since much of the work on that possibility [5] was done at Fermilab, if SIMPs were to be detected at Fermilab and we encourage those with influence in the collaborations to explore vigorously that possibility. Finally, we note that we proceed without committing to a specific SIMP model. We parameterize the experimental predictions in terms of the two parameters $\sigma_{S N}$ and $M_{S}$.

\section{SIMPs in Nuclei}

We know a fair amount about SIMP binding in nuclei from the phenomenology of hyper-fragments. See, for example, Povh [8] for a readable review. Based on that experience, we can write for the binding $B$ of the SIMP in a nucleus A the relation:

$$
B=\left|V_{S N}\right|-\pi^{2} /\left(2 \mu R^{2}\right)
$$

where $\mu$ is the reduced mass of the $\mathrm{S}-\mathrm{A}$ system, $\mathrm{R}$ is the radius of the nucleus $\mathrm{A}$, and $\mathrm{V}$ is the $\mathrm{S}-\mathrm{N}$ potential averaged over the volume of the nucleus $\mathrm{X}$. We expect the low energy potential, $V_{S N}$, to be always attractive. This is true if exchange of vacuum quantum numbers dominates. We assume this to be the case, and have not found a model to the contrary. Under this assumption, the SIMP can be bound in a nucleus for which $\mu$ and $R^{2} \sim A^{2 / 3}$ are large enough to make the kinetic energy less than the (average) magnitude of the attractive potential.

From equation (1) we see that the best chance of finding SIMPs is to search in high Z (large) nuclei which minimize the kinetic energy term. Capture by light

\footnotetext{
${ }_{\ddagger}$ We are grateful to Professor Ephraim Fischbach for keeping us informed as to progress on this crucial experiment.
} 
elements at the time of cosmic nucleosynthesis has been studied in ref. [9]. Atomic Mass Spectrometer (AMS) searches to date are reviewed in the careful study of Hemmick et al., [10] where one learns the somewhat surprising fact that previous searches have only been conducted up to sodium $(Z=11, A=23)$. This makes the current Purdue AMS experiment particularly exciting. They are looking in gold $(Z=79, A=100)$. At the risk, however, of sounding greedy, we would be interested in anyone with AMS equipment, a supply of Lawrencium $(Z=103, A=262)$, and an affinity for maximal experiments. However, as will become clear below, we do not want any such calls from interested parties to be collect.

How big is the potential $V_{X N}$ ? We take this as a parameter, but we can put an approximate LOWER bound on it from the requirement that primordial $S$ and $\bar{S}$, left over from the early universe, not overclose the universe so that it couldn't have continued expanding until today (late 1999). The classic book of Kolb and Turner [11] tells us that the number density of primordial SIMPs behaves as

$$
n_{S} \sim\left(M_{S} \sigma_{S \bar{S}}\right)^{-1}
$$

Equation (2) says that too small an annihilation cross section means too many SIMPs will be left over from the early universe, and Kolb and Turner collect together the numerical recipes for computing how small is too small. We still need, however, to relate the annihilation cross section, $\sigma_{S \bar{S}}$ to the SIMP-nucleon cross section, $\sigma_{S N}$ and to the $S-N$ potential in Equation (1). We make the simple ansatz

$$
\begin{gathered}
V_{S N}=V_{N N}\left(\sigma_{S N} / \sigma_{N N}\right)^{1 / 2} \\
\sigma_{S N}^{2}=\beta \sigma_{N N} \sigma_{S \bar{S}}
\end{gathered}
$$

where $\beta$ should be on the order of one. Note that $V_{S N}$ goes as $\beta^{1 / 4}$ so that our results for binding will not be highly dependent on the precision of Equation (3).

Now that we know, for each point in the $M_{S}, \sigma_{S N}$ parameter space, the primordial $\mathrm{S}$ abundance and the binding energy in nuclei, we are almost ready to compute for our friends at Purdue, the abundance of anomalous gold-gold with a SIMP bound in it. First, however, we need a scenario for how the SIMPs get bound into the gold. Our picture is as follows:

- We assume that the ratio of SIMPs to protons in the galaxy is the same as the cosmic ratio, but that most of the SIMPs are in the galactic halo (i.e., that their density distribution is $\rho \sim R^{-2}$ ), not in stars. We can then calculate the SIMP flux on the Earth, since we know that the Earth is traveling through the galaxy with a velocity of about $200 \mathrm{~km} / \mathrm{s}$ which not too different from the galactic virial velocity.

- We assume that when the SIMP hits the Earth, it is slowed by scattering with all nucleons and nuclei at a rate determined by $\sigma_{S N}$, but can only be captured by a nucleus that is large enough. 
- Gold must compete, for SIMP capture, with the most abundant nuclei large enough to bind the SIMP. Our comparative estimates use, as the most abundant elements:, aluminum $(A=27)$, barium $(A=137)$, and lead $(A=206)$.

Our procedure is then as follows:

- We chose values for $M_{S}$ and $\sigma_{S N}$ and then determine whether, for that point in parameter space, there is binding in gold.

- Assuming that there is binding, we then determine (a) the mean free path in Earth from the galactic virial velocity and $\sigma_{S N}$, and (b) which of the 3 elements above is gold's chief competitor for SIMP capture.

- From the ratio of the abundance of gold to its chief competitor, the mean free path, and the average density of Earth, we then compute the chance of a particular gold nucleus within a mean free path to capture an incident SIMP. Multiplying by the flux (see above) of SIMPs and the time for which the sample being put in the AMS target has been exposed (which is why we don't want collect calls from those long in Lawrencium) gives us the fraction of gold nuclei in the sample that should have a SIMP if they exist at that point in parameter space.

Finally, we assume that the exposure time is 10 million years because there are regions that are geologically inactive over such periods and have had for example "placer" gold in the beds of streams for a longer period than that

The results are shown in the table. It gives $\log _{10}$ of the ratio of normal to anomalous gold nuclei. The dashes indicate parameter values for which there is either no binding in gold or overclosure of the universe. One sees that smaller values of $\sigma_{S N}$ give larger ratios of anomalous to normal gold. This is because smaller values imply that only lead has a nucleus large enough to compete with gold for SIMP capture and because the smaller cross section means more primordial abundance. The important thing to take away from hours of table study is the fact that the relative abundance entries are all considerably higher (for anomalous to normal) than the limits of $10^{-20}$ that have been set in AMS work on some of the light elements. This provides reason to expect that, if the SIMPs are there, the Purdue AMS people will find them.

\section{SIMPs at Fermilab}

Next we consider $S \bar{S}$ production at the Tevatron. Since we are talking neutral SIMPs, we expect little or no signal in the central tracker and in the electromagnetic calorimeter. However, in the hadron calorimeter, we expect to detect SIMP signals if

\footnotetext{
$\S$ We appreciate conversations with Professor E. T. Herrin on searching for old exposed gold.

IPurdue is, we believe, taking collect calls from people who have pieces of gold with such provenance; they give all but a small fraction of a mole back at the end of the run.
} 


\begin{tabular}{||c||c|c|c|c|c|c|c|c|c|c|c|c|}
\hline \hline & 0.0005 & 0.0042 & 0.012 & 0.032 & 0.25 & 0.69 & 1.9 & 5.3 & 15 & 41 & 110 & 860 \\
\hline \hline 1.0 & - & - & - & - & - & - & - & 6.3 & 8.3 & 8.7 & 12.5 & 13.4 \\
\hline 1.6 & - & - & - & - & - & - & 6.1 & 8.1 & 8.5 & 12.3 & 12.7 & 13.6 \\
\hline 2.7 & - & - & - & - & - & 5.9 & 7.9 & 8.3 & 12.1 & 12.5 & 12.9 & 13.8 \\
\hline 4.3 & - & - & - & - & 5.7 & 7.7 & 8.1 & 11.1 & 12.3 & 12.7 & 13.1 & 14.0 \\
\hline 7.1 & - & - & - & - & 7.5 & 7.9 & 10.9 & 12.1 & 12.5 & 12.9 & 13.4 & 14.2 \\
\hline 12 & - & - & - & 5.6 & 8.1 & 8.5 & 12.2 & 12.7 & 13.1 & 13.5 & 13.9 & 14.8 \\
\hline 19 & - & - & - & 7.5 & 8.3 & 11.3 & 12.5 & 12.9 & 13.3 & 13.8 & 14.2 & 15.0 \\
\hline 31 & - & - & 7.4 & 7.8 & 8.6 & 12.4 & 12.8 & 13.2 & 13.6 & 14.1 & 14.5 & 15.3 \\
\hline 50 & - & 5.7 & 7.7 & 8.1 & 11.5 & 12.7 & 13.1 & 13.6 & 14.0 & 14.4 & 14.8 & 15.7 \\
\hline 81 & 5.7 & 7.7 & 8.1 & 8.5 & 11.9 & 13.1 & 13.5 & 14.0 & 14.4 & 14.8 & 15.2 & 16.1 \\
\hline 132 & 7.7 & 8.1 & 8.5 & 8.9 & 12.2 & 13.5 & 13.9 & 14.3 & 14.7 & 15.2 & 15.6 & 16.4 \\
\hline 220 & 8.0 & 8.4 & 8.9 & 9.3 & 12.6 & 13.9 & 14.3 & 14.7 & 15.1 & 15.5 & 16.0 & 16.8 \\
\hline 350 & 8.4 & 8.8 & 9.3 & 9.7 & 13.8 & 14.3 & 14.7 & 15.1 & 15.5 & 15.9 & 16.4 & 17.2 \\
\hline 570 & 8.8 & 9.2 & 9.7 & 10.1 & 14.3 & 14.7 & 15.1 & 15.5 & 15.9 & 16.4 & 16.8 & 17.6 \\
\hline 930 & 9.3 & 9.7 & 10.1 & 10.5 & 14.7 & 15.1 & 15.5 & 16.0 & 16.4 & 16.8 & 17.2 & 18.1 \\
\hline \hline
\end{tabular}

Table 1. $M_{X}$ (vertical) is in units of $\mathrm{GeV}$, and $\sigma_{X N}$ (horizontal) is in units of $m b$. Table entries are $\log _{10}(1 / f)$, and the - indicates those cases for which $X$ does not bind at all.

$\sigma_{S N}$ is large enough. The detection of SIMPs is possible if one triggers on two back-toback hadron calorimeter showers, accompanied by little else. We will use $10 \mathrm{GeV}$ for the minimum size showers for which such triggering might be done. Our task now is to determine:

- For what values of $\left\{M_{S}, \sigma_{S}\right\}$ will the SIMP interact in the steel plates of the hadron calorimeter?

- For what values of these parameters will we get calorimeter showers greater than $10 \mathrm{GeV}$ or more?

- Can one recognize a SIMP shower if one sees one?

- How many such events should we expect?

First we look at the region of parameter space for which there will be interaction. The minimum annihilation cross section permitted by the cosmology argument is $\sim 3 \times 10^{-13}$ barns, which corresponds through Equation (3) to about a microbarn for the $\mathrm{S}-\mathrm{N}$ cross section. SIMPs with such small cross sections won't shower in 1 meter 


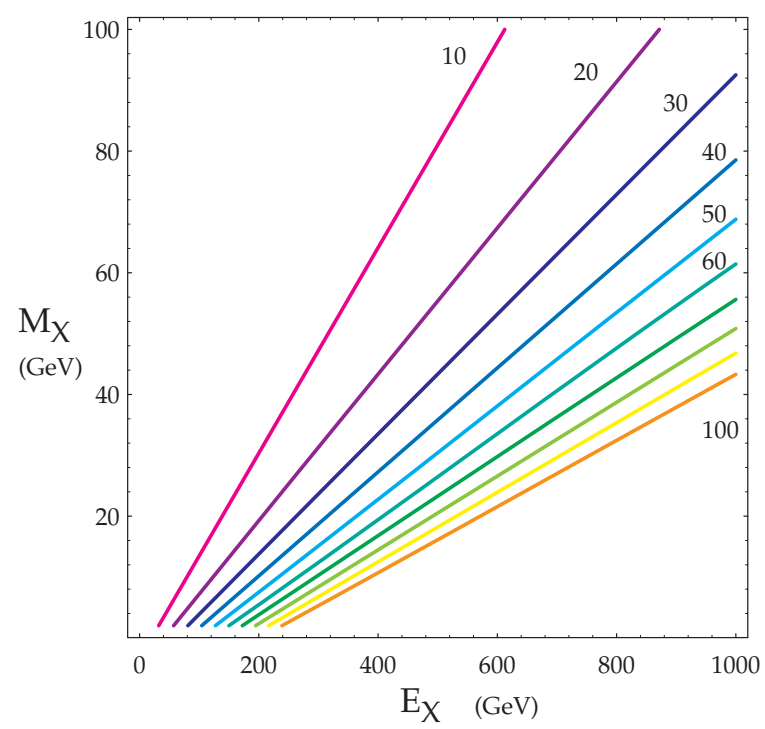

Figure 1. Contours for energy loss as a function of $\left\{M_{X}, E_{X}\right\}$. The contours displayed are in steps of $10 \mathrm{GeV}$.

of steel, but for a higher cross section of a few millibarns, we would expect 10 or more interactions with the $10^{27}$ nucleons $/ \mathrm{cm}^{2}$ in the 1 meter.

To estimate the energy we expect in a shower resulting from a SIMP interaction in the steel plates of a hadron calorimeter we use a cosmic ray rule of thumb kindly provided by G. Yodh the center of mass energy goes into inelasticity. In the figure, we give the (laboratory) energy released in the calorimeter as we vary the mass and energy of the SIMP; the straight lines are constant shower energies. One sees that the bigger the SIMP lab energy, the greater a SIMP mass will result in a given shower energy.

Consider now the question of whether we would recognize a SIMP shower if we saw one. The background for SIMP showers would likely be neutron showers and $K$ decays. The distinguishing feature would be shower opening angle. A pion moving transverse in the c.m. system would have a lab angle given by $\tan \theta=1 / \gamma$. Comparing the angle for a SIMP with that from a neutron of the same energy, the SIMP shower should be wider by roughly the ratio of the masses.

Finally, we turn to the number of SIMP pairs the Tevatron might produce. We scale the (known) production rate of jets by the ratio of the S-N cross section to that of Meson-N, which we take to be on the order of 30 millibarns. So long as the SIMP energy is a few times its mass, we don't worry about phase space suppression. We assume conservatively a cross section of about $3 p b$ for any one parton in the region

"This useful approximation from Professor Gaurang Yodh made the whole trip to Paris (where the conversation took place) well worthwhile (and the food was OK too). 
$E>200 \mathrm{GeV}$. This implies about 6000 events in Run II. The estimate of [5] is that the Nucleon-UHECR cross section needs to be over a tenth the Meson-Nucleon cross section, so we estimate 600 events in the Tevatron run if SIMPs are the explanation for the UHECR events.

\section{Summary}

For the Table we see that there is SIMP binding in gold for $M_{S}^{2} \sigma_{S N}>5 m b G e V^{2}$, and that AMS experiments sensitive to one part in $10^{20}$ can detect the existence of SIMPs of mass less than a TeV, while the region of interest for explaining UHECRs can be explored with a sensitivity of one part in $10^{16}$ or less. Looking for SIMPs at the Tevatron is more difficult, but over half the region of interest for explaining UHECRs could be searched in the upcoming Run II by looking for (wide) back to back jets with no signal in the central tracker or EM calorimeter. Interested people are urged to send any gold in their possession for which they can prove $10^{7}$ (or more) years of exposure to Purdue. Almost all nuclei will be returned intact**.

We thank D. Berley, K. Brockett, K. De, D. Dicus, M.A. Doncheski, R. Ellsworth, G. Farrar, E. T. Herrin, D. Rosenbaum, R. Scalise, and G. Yodh. The work of RNM has been supported by the National Science Foundation grant under no. PHY-9802551. The work of Olness, Stroynowski, and Teplitz is supported by DOE.

\section{REFERENCES}

1. C. Dover, T, Gaisser, and G. Steigman, Phys. Rev. Lett. 42, 1117 (1979).

2. S. Wolfram, Phys. Lett. 82B, 65 (1979).

3. G. Starkman et al., Phys. Rev. D41 2388 (1990).

4. H. Baer, K. Cheung and J. F. Gunion, Phys. Rev. D59, 075002 (1999); S. Raby and K. Tobe, Nucl. Phys. B539, 3 (1999); A. Mafi and S. Raby, hep-ph/9912436, and references therein.

5. D. Chung, G. Farrar, and E. W. Kolb, Phys. Rev. D57, 4606 (1998) and I. F. M. Albequerque, G. Farrar, and E. W. Kolb, Phys. Rev. D59 015021 (1999).

6. R. N. Mohapatra, F. Olness, R. Stroynowski, and V. L. Teplitz, Phys. Rev. D60, 115013 (1999).

7. R. N. Mohapatra and V. L. Teplitz, Phys. Rev. Lett. 81, 3079 (1998).

8. Z. Povh, Ann. Rev. Nuc. Sci. 28, 1 (1978).

9. D. A. Dicus and V. L. Teplitz, Phys. Rev. Lett. 44, 218 (1980).

10. T. K. Hemmick et al., Phys. Rev. D41 2074 (1990).

11. E. W. Kolb and M. Turner, The Early Universe (Addison Wesley, Reading, MA, 1990).

\footnotetext{
** Sorry, but neither Purdue, SMU, or Maryland can be responsible for nuclei lost or damaged in transit (by either AMS or Fedex)
} 